\title{
Application of Thermoresponsive PNIPAAM-b-PAMPTMA Diblock Copolymers in siRNA Delivery
}

Ana M. Cardoso, ${ }^{\dagger \dagger}$ M. Teresa Calejo, ${ }^{\S}$ Catarina M. Morais, ${ }^{\dagger \dagger}$ Ana L. Cardoso, ${ }^{\dagger}$ Rita Cruz, Kaizheng Zhu, " Maria C. Pedroso de Lima, ${ }^{\dagger, \ddagger}$ Amália S. Jurado, ${ }^{* \dagger, \dagger}$ and Bo Nyström*,\|

${ }^{\dagger} \mathrm{CNC}-$ Centre for Neurosciences and Cell Biology, University of Coimbra, 3004-517 Coimbra, Portugal

${ }^{\ddagger}$ Department of Life Sciences, University of Coimbra, 3004-517 Coimbra, Portugal

${ }^{\S}$ Department of Electronics and Communications Engineering, Tampere University of Technology, 33720 Tampere, Finland

"Department of Chemistry, University of Oslo, 0313 Oslo, Norway
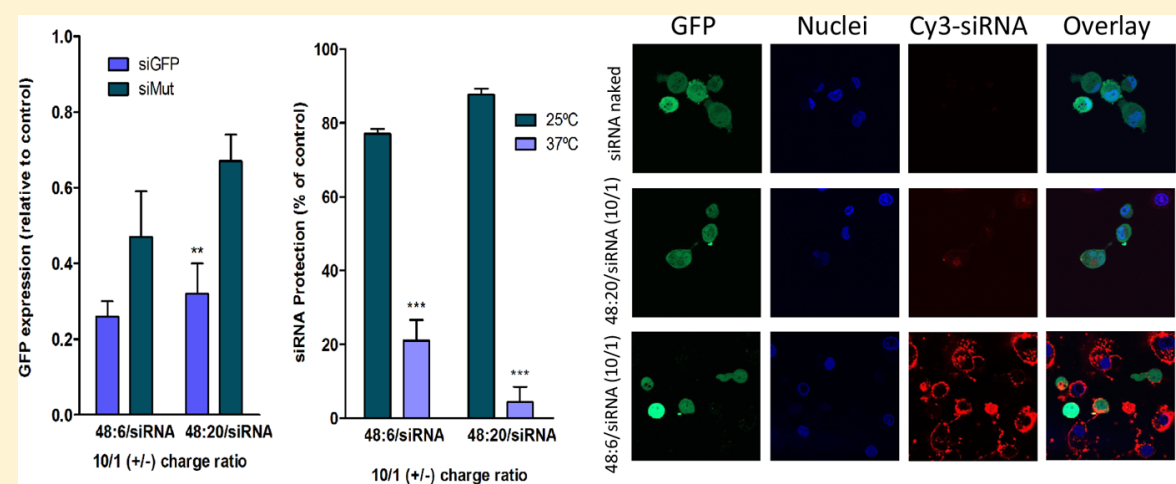

ABSTRACT: Gene knockdown has emerged as an important tool for cancer gene therapy as well as for viral infections and dominantly inherited genetic disorders. The generation of suitable siRNA delivery systems poses some challenges, namely, to avoid nuclease degradation, to surpass the cytoplasmic membrane, and to release the nucleic acids into the cytosol. Aiming at evaluating the ability of thermoresponsive block copolymers formed by units of $\mathrm{N}$-isopropylacrylamide and of (3acrylamidopropyl)trimethylammonium chloride to efficiently deliver siRNAs, an extensive study was performed with four different copolymers using a human fibrosarcoma cell line as cell model. The silencing ability and cytotoxicity of the generated copolymer-based siRNA delivery systems were found to be dependent on the cloud point of the polymer, which corresponds to the transition temperature at which the aggregation or precipitation of the polymer molecules becomes thermodynamically more favorable than their solubilization. In the present study, a system capable of delivering siRNAs efficiently, specifically and without presenting relevant cytotoxicity, even in the presence of serum, was developed. Confocal fluorescence experiments showed that the ability of the generated systems to silence the target gene is related to some extent to nucleic acid internalization, being also dependent on polymer/siRNA dissociation at $37{ }^{\circ} \mathrm{C}$. Thus, a delicate balance between nucleic acid internalization and intracellular release must be met in order to reach an ideal knockdown efficiency. The special features and potential for manipulation of the $\mathrm{N}$-isopropylacrylamide-based copolymers make them suitable materials for the design and synthesis of new and promising siRNA delivery systems.

KEYWORDS: siRNA delivery, nonviral vector, cancer gene therapy, thermoresponsive copolymer, polyplex, cloud point

\section{INTRODUCTION}

RNA interference (RNAi) is a powerful gene silencing process that holds a great therapeutic potential in several diseases, such as cancer. ${ }^{1}$ Small interfering RNAs (siRNAs), first described in 2001 as promoting effective RNA interference in mammalian cells without inducing interferon immunological response, ${ }^{2}$ are short double stranded RNA (dsRNA) molecules (less than 30 nucleotides in length) that mediate post-transcriptional silencing of target genes through degradation of their mRNAs. ${ }^{3}$ This process is highly efficient and sequencedependent, thus conferring specificity to the silencing mechanism. In Nature, siRNAs are produced as an antiviral defense, silencing mobile genetic elements resulting from viral infections. For therapeutic purposes, siRNAs have been delivered into cells using adequate carrier systems able to overcome membrane barriers and to release the nucleic acids into the cytosol, in order to allow their loading into the RNAinduced silencing complex (RISC) and the consequent specific mRNA knockdown. ${ }^{3}$ Several different nonviral delivery

Received: August 25, 2013

Revised: December 17, 2013

Accepted: January 15, 2014

Published: January 15, 2014 
systems, including cationic lipids, peptides, and polymers, have been developed to introduce siRNAs into the cell cytoplasm, where they are active. ${ }^{4-7}$

Some polymeric units, such as $\mathrm{N}$-isopropylacrylamide (NIPAAM), are thermoresponsive and display a nonlinear relationship between solubility and temperature, exhibiting a sharp thermotropic transition between a soluble and an insoluble state. ${ }^{8}$ The phase transition temperature, known as cloud point or lower critical solution temperature (LCST), represents a transition from a hydrated form (due to the formation of water-polymer hydrogen bonds) to a nonhydrated form of the polymers, in which the disruption of the hydrogen bonds is entropically favored, leading to collapse or macroscopic phase separation of the polymers depending on the polymer concentration. ${ }^{9}$ Since the cloud point of NIPAAM was known, in $1992,{ }^{8}$ homopolymers formed by NIPAAM units or copolymers designed to exhibit different combinations and ratios between NIPAAM and other different units, have been extensively studied for biomedical applications, including controlled delivery of therapeutic molecules and tissue engineering. ${ }^{10}$ One of the reasons why the polymers containing NIPAAM have gained increasing interest in therapeutic applications resides on the possibility of modulating their thermoresponsiveness by varying several parameters, such as salt concentration, $\mathrm{pH}$, and composition or relative abundance of polymer components. ${ }^{11,12}$

A polymer composed of several NIPAAM units, that is a poly-NIPAAM (PNIPAAM), hydrophobically aggregates at temperatures above $32{ }^{\circ} \mathrm{C}$, depending on concentration and molecular weight, ${ }^{12}$ and this proximity to human body temperature makes it particularly interesting for pharmaceutical and biomedical applications. ${ }^{13}$ Importantly, by copolymerization with other hydrophilic or hydrophobic blocks, one can increase or decrease the phase transition temperature of the polymer, for specific applications. ${ }^{14}$ For instance, copolymerization with hydrophilic blocks to produce polymers with a cloud point close to $37{ }^{\circ} \mathrm{C}$ has revealed to be particularly advantageous since these molecules are sensitive to the physiological temperature, thus changing their structure when incorporated in the human organism. Therefore, complexes of these copolymers with nucleic acids, formed at temperatures below the polymer cloud point, are expected to display the ability to release the complexed molecule into human cells or body fluids due to a collapse of the polymeric structure at the physiological temperature. ${ }^{10}$ In this context, the addition of a cationic unit, such as (3-acrylamidopropyl)trimethylammonium chloride (AMPTMA), to the NIPAAM structure was envisaged to accomplish two purposes: to increase the cloud point of the polymer $^{10,12}$ and to confer positive charges to the overall molecule. ${ }^{15}$ The addition of cationic units to hydrophobic polymers has been reported as making the polymer more prone to efficiently complex negatively charged biomolecules such as nucleic acids and to interact with negatively charged cell membranes. ${ }^{15}$ This approach has been applied to design new copolymer-based plasmid DNA delivery systems, creating molecular combinations with delivery competence that none of the individual components of the copolymer exhibit due to cytotoxicity or impairment of specific steps in the delivery process, such as cellular internalization and endosomal escape. The main objective of combining two distinct monomers in the copolymer is to ally advantageous properties of each one and to minimize their deficiencies in order to accomplish an effective delivery. ${ }^{16}$
In this work, four diblock copolymers were synthesized as poly $(N \text {-iso propylacrylamide })_{n}-b l o c k$-poly ( (3acrylamidopropyl)trimethylammonium chloride $)_{m}$, abbreviated herein as PNIPAAM ${ }_{n}-b$-PAMPTMA $(+)_{m}$, or $n: m(n=48$ or 65 and $m=6,10$, or 20), which exhibit different cloud points and molecular weights. These block copolymers, which have already shown to be promising candidates as DNA nonviral carriers, ${ }^{17}$ were used in the present work to investigate their ability to efficiently deliver siRNA to HT1080 cells (a human fibrosarcoma cell line) in the absence and presence of serum. To the best of our knowledge, only one study has addressed the generation of a PNIPAAM-containing system for siRNA delivery, ${ }^{18}$ which was composed of quantum dots cografted with a cell penetrating peptide. Our results show that nucleic acid protection conferred by the polymers, associated with their ability to mediate cell internalization of the siRNAs and the polymer/siRNA dissociation, is responsible for silencing a target protein.

These data shed light on the great variety of existing possibilities to design new and more efficient siRNA delivery systems, with the ultimate goal of developing a useful tool to modulate gene expression with implications in several fields, such as functional genomics, drug validation, and even transgenic design. ${ }^{19-22}$

\section{EXPERIMENTAL SECTION}

Materials. The anti-GFP siRNA (5'-GCAAGCUGACCCUGAAGUUCAU-3') and Cy3-labeled nonspecific siRNA sequence were purchased from Ambion (Austin, TX, USA). The nonsilencing siRNA sequence used as control was obtained from Dharmacon (Lafayette, CO, USA). All the other chemicals were of the highest grade.

Charged Block Copolymers. The cationic diblock copolymers poly $(N \text {-isopropylacrylamide })_{n}$-block-poly $((3-$ acrylamidopropyl)trimethylammonium chloride) ${ }_{m}$ (PNIPAAM $_{n}-b$-PAMPTMA $\left.(+)_{m}\right)$ with $n=48$ or 65 and $m=6$, 10 , or 20 (Figure 1) were synthesized via a simple one-pot

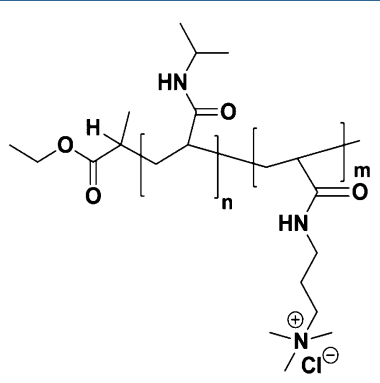

Figure 1. Schematic representation of the chemical structure of the PNIPAAM $_{n}-b$-PAMPTMA $(+)_{m}$ diblock copolymers $(n=48$ or 65 and $m=6,10$, or 20$)$.

atom transfer radical polymerization (ATRP) procedure, as previously reported. $^{23,24}$ The chemical structure of the diblock copolymers used in this study is illustrated in Figure 1, and their cloud point values and molecular weights are presented in Table 1.

Cells. HT1080 cells (a human fibrosarcoma cell line that was stably transfected to express GFP endogenously) were maintained at $37{ }^{\circ} \mathrm{C}$, under $5 \% \mathrm{CO}_{2}$, in Dulbecco Modified Eagle low glucose (DMEM-LG; Sigma) supplemented with $10 \%(\mathrm{v} / \mathrm{v})$ heat-inactivated fetal bovine serum (FBS; Biochrom 


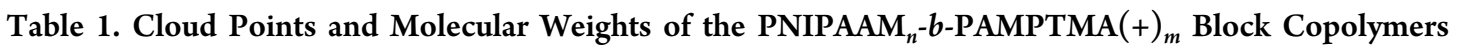

\begin{tabular}{|c|c|c|c|c|}
\hline polymer & $\begin{array}{c}\text { PNIPAAM }_{48}-b \text {-PAMPTMA }(+)_{6} \\
(48: 6)\end{array}$ & $\begin{array}{c}\text { PNIPAAM }_{48}-b \text {-PAMPTMA }(+)_{10} \\
(48: 10)\end{array}$ & $\begin{array}{c}\text { PNIPAAM }_{48}-b \text {-PAMPTMA }(+)_{20} \\
(48: 20)\end{array}$ & $\begin{array}{c}\text { PNIPAAM }_{65}-b \text {-PAMPTMA }(+)_{20} \\
(65: 20)\end{array}$ \\
\hline $\begin{array}{l}\text { cloud point } \\
\left({ }^{\circ} \mathrm{C}\right)^{a}\end{array}$ & 35 & 36 & 37 & 34 \\
\hline$M_{\mathrm{w}}(\mathrm{g} / \mathrm{mol})^{a}$ & 6770 & 7600 & 9690 & 11600 \\
\hline
\end{tabular}

$\mathrm{KG}$ ), and with 100 units of penicillin and $100 \mu \mathrm{g}$ of streptomycin (Sigma) per mL.

Preparation of Polymer/siRNA Complexes. Polymers were dissolved in cell culture medium (OptiMEM or DMEMLG) and gently mixed with siRNA targeting GFP (50 nM/ well), at the desired polymer/siRNA $(+/-)$ charge ratios. The mixtures were further incubated for $20 \mathrm{~min}$ at room temperature. Polymer/siRNA complexes were used immediately after being prepared.

Determination of the Hydrodynamic Diameter of Polymer/siRNA Complexes. Polyplexes were characterized with respect to their hydrodynamic diameter in HBS buffer, at 25 and $37{ }^{\circ} \mathrm{C}$, by photon correlation spectroscopy-based technique (PCS) using a Coulter N4 Plus (Coulter Corporation, Miami, FL, USA). PCS uses photon correlation spectroscopy of scattered laser light to determine its timedependent fluctuations resulting from the Brownian motion of particles in suspension. The light intensity scattered at a given angle is detected by a detector (at fixed angle of $90^{\circ}$ ) whose output current is passed to a photocorrelator, which analyses time dependence, determining the rate of diffusion or Brownian motion of the particles and hence their hydrodynamic diameter. ${ }^{25}$ Complexes were prepared immediately before analysis. All complexes showed a monomodal hydrodynamic diameter distribution.

Evaluation of Cell Viability. Cell viability was assessed under the different experimental conditions by a modified Alamar blue assay, as described previously. ${ }^{26}$ Briefly, $48 \mathrm{~h}$ posttransfection, the cells were incubated with DMEM-LG (10\% serum) containing $10 \%(\mathrm{v} / \mathrm{v})$ resazurin dye. After $1 \mathrm{~h}$ incubation at $37{ }^{\circ} \mathrm{C}$, the absorbance of the medium was measured at 570 and $600 \mathrm{~nm}$ (supplier indication). Cell viability was calculated as a percentage of the nontransfected control cells, according to eq 1 :

$$
\begin{aligned}
& \text { cell viability (\% of control) } \\
& \quad=\left[\left(A_{570}-A_{600}\right) /\left(A^{\prime}{ }_{570}-A^{\prime}{ }_{600}\right)\right] \times 100
\end{aligned}
$$

where $A_{570}$ and $A_{600}$ are the absorbances of the samples, and $A^{\prime}{ }_{570}$ and $A^{\prime}{ }_{600}$ are those of the control (nontreated cells), at the indicated wavelengths.

Analysis of GFP Silencing. HT1080 cells $\left(1.0 \times 10^{5}\right.$ cells/ well) were seeded in 12 -well plates. Following overnight culture, the cells were incubated with the different complexes (50 nM of siRNA per well) at $37^{\circ} \mathrm{C}$ for $4 \mathrm{~h}$, in OptiMEM (no serum) or DMEM-LG (10\% serum) media. After this period, the medium was replaced with fresh complete medium containing $10 \%(\mathrm{v} / \mathrm{v})$ FBS and antibiotics, and the cells were further incubated for $44 \mathrm{~h}$ to allow gene expression. Parallel experiments were performed with a control siRNA (nontarget siRNA or siRNA mut). The knockdown efficiency mediated by the different complexes was evaluated by analyzing GFP silencing by flow cytometry. Briefly, $48 \mathrm{~h}$ after transfection, the cells were washed once with PBS and detached with trypsin (10 $\mathrm{min}$ at $37^{\circ} \mathrm{C}$ ). The cells were further washed three times by centrifugation (5 min, $1000 \mathrm{rpm}$ ) in ice-cold PBS and immediately analyzed, using a Becton Dickinson FACSCalibur flow cytometer (BD Biosciences, San Jose, CA, USA). Data obtained were analyzed using CellQuest software. To discriminate between viable and dead cells and to exclude doublets, cells were appropriately gated by forward/side scatter and pulse width from a total of 10000 events. The FITC bandpass filter was used in emission detection. GFP silencing was expressed as geometric mean fluorescence intensity with respect to control (nontreated) cells.

Analysis of siRNA Internalization and Intracellular Distribution. HT1080 cells $\left(0.2 \times 10^{5}\right.$ cells/well $)$ were plated in 8-well chambered coverslips (Lab-Tek II Chamber Slide System Nunc). Following overnight culture, the cells were incubated, for $4 \mathrm{~h}$, with the different polymer/siRNA delivery systems formulated with noncoding Cy3-labeled siRNAs (50 $\mathrm{nM}$ ), in OptiMEM medium. After this period, cells were washed twice with PBS and incubated with Hoechst 33342 dye $(1 \mu \mathrm{g} / \mathrm{mL}$, Molecular Probes, Eugene, OR). Cells were then washed twice with PBS and directly observed in the chambers in $0.5 \mathrm{~mL}$ of PBS. Fluorescence distribution inside cells was analyzed under a Zeiss Axiovert $200 \mathrm{M}$ confocal microscope (Carl Zeiss, Oberk) without fixation, using the $60 \times$ oil immersion objective.

Analysis of siRNA Protection. Complexes containing 14 pmol of siRNA prepared in a total volume of $100 \mu \mathrm{L}$ of HBS were allowed to incubate for $20 \mathrm{~min}$ at room temperature and then transferred to a 96-well (blackwalled) plate (Corning, NY, USA). A total of $100 \mu \mathrm{L}$ of PicoGreen dye (Molecular Probes, Eugene, OR), upon 1:200 dilution in HBS buffer (according to the manufacturer's instructions), was added to each well. The fluorescence of PicoGreen, directly proportional to the amount of accessible/free siRNA, was monitored in a Spex Fluorolog Spectrometer at 25 and $37{ }^{\circ} \mathrm{C}$. The excitation and emission wavelengths were set at 485 and $520 \mathrm{~nm}$, respectively. The amount of siRNA protected by the cationic polymers was calculated as a percentage of a control containing only a PicoGreen solution, according to eq 2 :

$$
P_{\text {siRNA }}=1-\left[\left(F-F_{100}\right) /\left(F_{0}-F_{100}\right)\right]
$$

where $F$ is the fluorescence measured after adding the complexes to the PicoGreen solution, $F_{0}$ is the maximum fluorescence of a positive control, which accounts for $0 \%$ of siRNA protection, and $F_{100}$ is the minimum fluorescence of a negative control taken as $100 \%$ of protection. For the positive control, free siRNA, in the same amount as that associated with the complexes, was used and a PicoGreen solution without siRNA was used as the negative control.

Statistical Analysis. Data are presented as mean \pm SD. The significance of the results was statistically analyzed by a one-way analysis of variance (ANOVA) with Tukey's multiple pairwise comparison, unless stated otherwise. Statistical significance was set at $p<0.05$. 


\section{RESULTS}

Hydrodynamic Diameter of the Polymer/siRNA Complexes. Because of the temperature-dependent structural properties displayed by the polymers used in this study, the average diameter of their complexes with siRNA was evaluated at 25 and $37^{\circ} \mathrm{C}$. As shown in Table 2, polyplexes prepared

Table 2. Mean Hydrodynamic Diameter of the Polymer/ siRNA Complexes Obtained at 25 and $37^{\circ} \mathrm{C}$

\begin{tabular}{|c|c|c|c|}
\hline \multirow[b]{2}{*}{$\mathrm{n}: \mathrm{m}$} & \multirow[b]{2}{*}{$(+/-)$ charge ratio } & \multicolumn{2}{|c|}{ hydrodynamic diameter $(\mathrm{nm})^{a}$} \\
\hline & & $25{ }^{\circ} \mathrm{C}$ & $37^{\circ} \mathrm{C}$ \\
\hline \multirow[t]{2}{*}{$48: 6$} & $2 / 1$ & $179 \pm 34$ & $134 \pm 63$ \\
\hline & $10 / 1$ & $280 \pm 7$ & $309 \pm 78$ \\
\hline \multirow[t]{2}{*}{$48: 10$} & $2 / 1$ & $306 \pm 68$ & $217 \pm 38$ \\
\hline & $10 / 1$ & $336 \pm 12$ & $427 \pm 16$ \\
\hline \multirow[t]{2}{*}{$48: 20$} & $2 / 1$ & $820 \pm 82$ & $264 \pm 51^{* * *}$ \\
\hline & $10 / 1$ & $583 \pm 23$ & $140 \pm 61^{* * *}$ \\
\hline \multirow[t]{2}{*}{$65: 20$} & $2 / 1$ & $299 \pm 25$ & $295 \pm 68$ \\
\hline & $10 / 1$ & $442 \pm 67$ & $420 \pm 52$ \\
\hline
\end{tabular}

${ }^{a}$ Results represent the mean \pm SD from three independent experiments. Data comparisons were performed between the hydrodynamic diameters of complex formulations measured at 25 and $37^{\circ} \mathrm{C}$ $(* * * p<0.001)$.

from $n: m$ 48:20 exhibited the largest hydrodynamic diameters at $25{ }^{\circ} \mathrm{C}$ (820 and $583 \mathrm{~nm}$, when prepared at $2 / 1$ and $10 / 1$ $(+/-)$ charge ratios, respectively). For the same length of polymer thermoresponsive moiety $(n=48)$, the increase in the number of cationic units (from 6 to 20) led to a progressive increase of polyplex hydrodynamic diameter. However, the length of the thermoresponsive component also influenced polyplex dimensions. Thus, for the same number of cationic units $(m=20)$, the increase of the thermoresponsive moiety length (from 48 to 65) promoted a decrease of polyplex hydrodynamic diameter.

Complexes exhibited at $37{ }^{\circ} \mathrm{C}$ the same diameter as that observed at $25{ }^{\circ} \mathrm{C}$, except for those generated from the $n: m$ 48:20 polymer, which showed a significant reduction in hydrodynamic diameter with increasing temperature (to 263 and $140 \mathrm{~nm}$ when prepared at $2 / 1$ and $10 / 1(+/-)$ charge ratios, respectively).

Cytotoxicity and Silencing Efficiency. The cytotoxicity and silencing efficiency of complexes (polyplexes) composed of siRNA targeting GFP and each of four PNIPAAM $_{n}-b$ PAMPTMA $(+)_{m}$ block copolymers with different cloud points were evaluated in HT1080 cells, both in the absence and presence of $10 \%$ serum.

In the absence of serum, the toxicity exerted by the polyplexes showed high dependence on the polymer/siRNA $(+/-)$ charge ratio (Figure $2 \mathrm{~A})$. At the lowest $(+/-)$ charge ratio tested $(2 / 1)$, only the $n: m$ 65:20 polymer-containing complexes exhibited some toxicity, inducing a decrease of $37 \%$ in cell viability. At the polymer/siRNA (+/-) charge ratios of $5 / 1$ and $10 / 1$, the complexes were toxic, causing a reduction of cell viability to values between $60 \%[n: m 48: 20$ at $10 / 1(+/-)$ charge ratio] and $30 \%$ [ $n: m$ 65:20 at 10/1 (+/-) charge ratio], except for the $n: m$ 48:20 polymer-containing complexes at the $5 / 1(+/-)$ charge ratio, which decreased the cell viability only to $84 \%$. The $n: m$ 48:20-based complexes were, therefore, the least toxic to the cells in the absence of serum. Comparatively, the cytotoxicity of the complexes in the presence of serum was in general less severe (Figure 2B). In this case, only for the highest (+/-) charge ratio tested (10/1), the complexes showed significant cytotoxicity. The most pronounced toxic effect was observed for the complexes containing the $n: m$ 65:20 polymer, the cell viability reaching values of $46 \%$ and $32 \%$ in the presence of complexes at $5 / 1$ and $10 / 1(+/-)$ charge ratios, respectively.

Regarding the silencing efficiency for cells incubated with the complexes in the absence of serum (Figure 2C), only the formulations that exhibited cytotoxicity displayed unspecific silencing. As observed, complexes prepared at the lowest (+/-) charge ratios (particularly at the $2 / 1$ charge ratio), at which they exhibited low or no toxic effects, showed high ability to specifically silence the target gene (black columns). Remarkably, the $n: m$ 48:6 polymer/siRNA complexes, prepared at the $2 / 1(+/-)$ charge ratio, were able to suppress gene expression in $36 \%$ in a specific manner. In the presence of serum, the silencing specificity of the complexes decreased, as deduced from the knockdown effect observed when the complexes were formulated with the nontarget siRNA (white bars in Figure $2 D)$. Despite this being the general scenario, it is important to note that complexes of the $n: m$ 48:20 polymer and siRNA, at the $10 / 1(+/-)$ charge ratio, presented a significantly lower unspecific silencing effect in the presence of serum than in its absence. In this case, for complexes formulated with the nontarget siRNA, GFP expression was reduced to $55 \%$ of the control in the absence of serum and to $67 \%$ in the presence of serum. However, as shown in Figure 2C,D, upon knockdown with the siRNA targeting GFP, protein expression was $34 \%$ and $32 \%$ of the control, in the presence and absence of serum, respectively, thus revealing an improvement of the performance of these complexes under conditions mimicking more closely those in vivo, regarding their specific effect on the target gene.

siRNA Internalization and Intracellular Distribution. Aiming at comparing the extent of cellular internalization and distribution of siRNA molecules mediated by polyplexes displaying different levels of specific silencing, complexes containing the polymers $n: m \quad 48: 6$ and $48: 20$ at polymer/ siRNA $(+/-)$ charge ratios of $2 / 1$ and $10 / 1$ were selected for confocal microscopy analysis. Figure 3 displays the siRNA internalization patterns obtained in HT1080 cells, in the absence of serum, for the different conditions tested. Both untreated cells (data not shown) and cells treated with naked siRNA (Figure 3A) were used as negative controls. In these two cases, no siRNA red fluorescence was noticeable, which is in accordance with the inability of the siRNA per se to enter the cells. Lipofectamine siRNAmax was used as a positive control (Figure 3B). As observed, this commercially available reagent was able to deliver siRNA to cells, which displayed some punctuated red fluorescence (round and intense red dots), most likely reflecting siRNA entrapment inside vesicles. However, some degree of siRNA dispersion throughout the cells could also be deduced from the observed diffuse red fluorescence. The $n: m$ 48:20 copolymer showed a poor ability to mediate the entry of siRNA into the cells after $4 \mathrm{~h}$ of cell incubation with the polyplexes, both at the $2 / 1$ and $10 / 1$ $(+/-)$ charge ratios (Figure 3C,D). However, the pattern of distribution of siRNAs delivered by the 10/1 (+/ ) charge ratio complexes was more diffuse than that of siRNAs delivered by lipofectamine siRNAmax, indicating that siRNA internalization mediated by the polymer-based complexes was followed by its release from intracellular vesicles into the cytosol. However, complexes containing the $n: m$ 48:6 copolymer were able to overload the cells with siRNA, at both charge ratios 

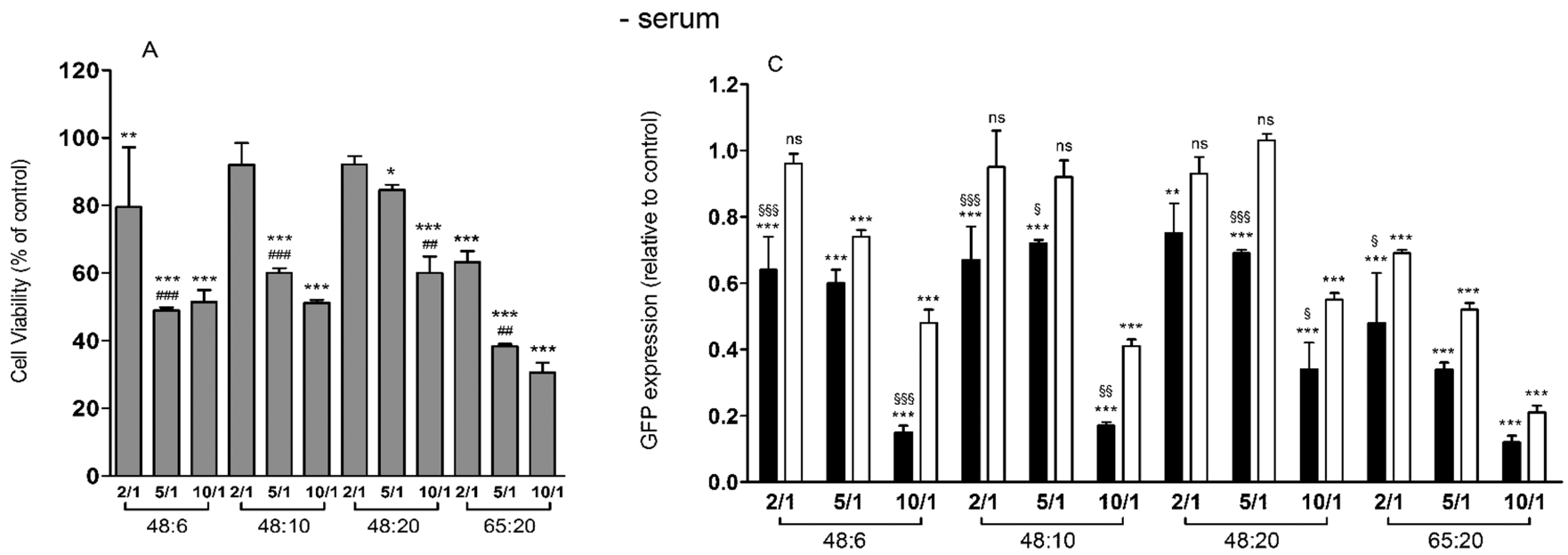

+ serum
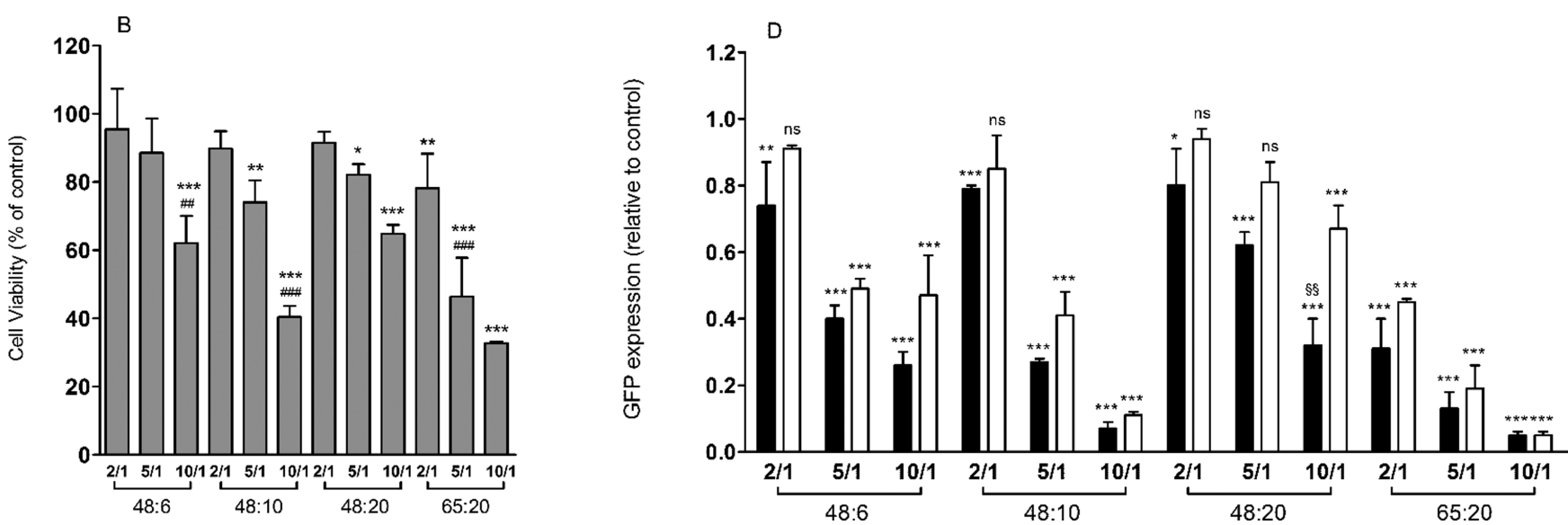

Figure 2. Cell viability $(A, B)$ and GFP expression $(C, D)$ promoted by polyplexes of PNIPAAM- $b$-PAMPTMA and siRNA in the absence (A,C) and in the presence of $10 \%$ serum (B,D) performed at $37^{\circ} \mathrm{C}$. Parallel experiments of GFP expression were performed with siRNA targeting GFP ( $\left.\mathbf{\square}\right)$ and a nontarget siRNA $(\square)$. The cell viability results are presented as a percentage of the control (nontreated cells, more than $98 \%$ of which are viable), taken as $100 \%$. Results of GFP expression are presented with respect to GFP expression of a control (nontreated cells, $80 \%$ of which express GFP), taken as 1.0. The results represent the mean \pm SD obtained from triplicates of three independent experiments. The following pairwise comparisons were performed: cell viability or GFP expression observed in cells treated with each complex formulation were compared to the same parameters obtained in nontreated control cells $\left({ }^{*} p<0.01, *^{*} p<0.005\right.$, and $* * * p<0.0001$; Dunnett's multiple comparison test); each complex formulation was compared to the same complex formulation at the immediately precedent $(+/-)$ charge ratio ${ }^{\#} p<0.01$, ${ }^{\# \#} p<0.005$, and ${ }^{\# \#} p<$ 0.0001); GFP expression obtained with complexes containing siRNA targeting GFP was compared with that obtained with complexes containing the nontarget siRNA $\left({ }^{\S} p<0.01,{ }^{\S} p<0.005\right.$, and ${ }^{\S \S_{s}} p<0.0001$; ns, nonsignificant $)$.

tested, as deduced from the intense and widely distributed red fluorescence observed throughout the cytoplasm (Figure 3E,F), thus showing their efficacy to promote both siRNA cellular uptake and cytoplasmic delivery.

siRNA Protection Conferred by the Polymers. The ability of the generated copolymers to confer protection to the siRNA was assessed by Picogreen fluorescence at 25 and $37^{\circ} \mathrm{C}$ (Figure 4). This assay takes advantage of the probe fluorescence emission only occurring upon probe intercalation in the nucleic acid structure. Therefore, the fluorescence measured in this assay reflects the accessibility of the nucleic acid in solution.

As observed, all the polymers studied herein were able to efficiently shield the siRNA molecules at $25^{\circ} \mathrm{C}$, with protection levels between 70 and $87 \%$, over the range of the tested charge ratios. However, results obtained from parallel experiments performed at $37^{\circ} \mathrm{C}$ showed that the copolymers essentially lost the capacity to protect the carried siRNAs. As observed, the maximum protection (30\%) was attained for complexes produced from the $n: m$ 48:6 block copolymer and siRNA at the $(+/-)$ charge ratio of $2 / 1$. Surprisingly, at this temperature $\left(37^{\circ} \mathrm{C}\right)$, the polymers showed a tendency for decreased ability to protect the siRNA molecules with increasing $(+/-)$ charge ratio. In fact, it should be expected that increasing the relative amount of the cationic molecule would increase the wrapping of the siRNAs, leading to a more efficient shielding of the carried nucleic acid molecules. That behavior was particularly noticed for polyplexes prepared from the copolymer $n: m$ 48:20, while for the polyplexes based on $n: m$ 48:6, 48:10, and 65:20 copolymers the degree of protection did not vary significantly with the $(+/-)$ charge ratio.

\section{DISCUSSION}

The temperature dependence of the structural properties exhibited by PNIPAAM-based block copolymers has been extensively exploited in recent years, with the purpose of producing delivery systems with controlled release ability for pDNA and other therapeutic molecules. ${ }^{10}$ 

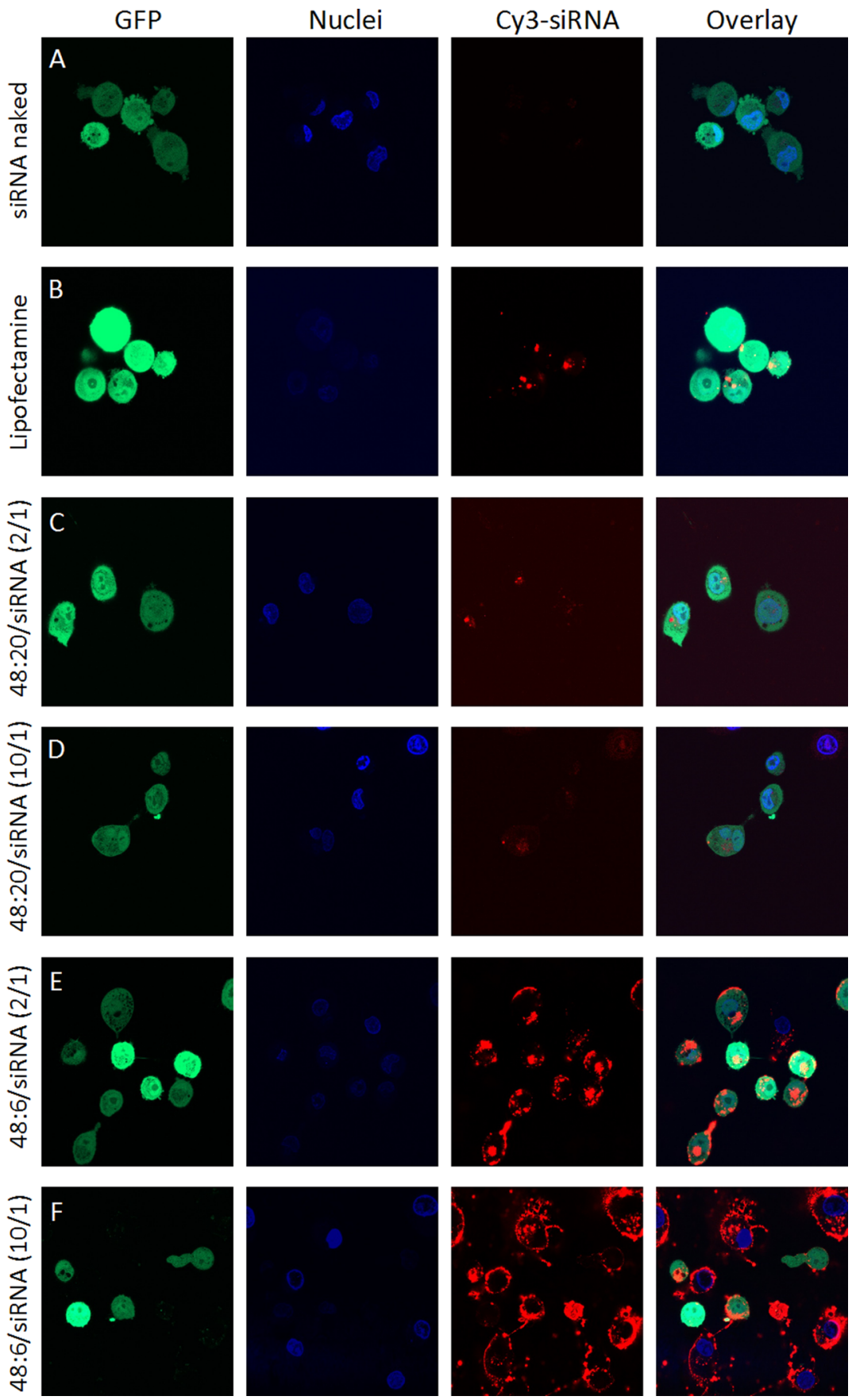

Figure 3. siRNA internalization mediated by different polymer-based systems at $37{ }^{\circ} \mathrm{C}$. HT1080 cells (green) were incubated with the different complexes containing $50 \mathrm{nM}$ nontarget Cy3-labeled siRNA (red). Cell nuclei were labeled with Hoechst 33342 dye (blue). Representative confocal microscopy images of each experimental condition (630X), taken after $4 \mathrm{~h}$ of cell incubation with the complexes are presented (A-F). Representative results of siRNA internalization in cells treated with lipofectamine siRNAmax/Cy3-siRNA (B), 48:20 polymer/Cy3-siRNA (C,D), and 48:6 polymer/Cy3-siRNA (E,F) complexes, at the indicated charge ratios, are presented. Results for cells treated with Cy3-siRNA only (siRNA naked) are shown as a control (A).

In this study, four cationic diblock copolymers differing in terms of the length of the thermoresponsive and the charged blocks were used. In a previous work, ${ }^{12,17}$ it was shown that the length and ratio between these blocks are determinant of the structural changes taking place as the temperature is increased, influencing the transfection efficiency. For a constant length of the PNIPAAM block, increasing length of the PAMPTMA $(+)$ block led to an increase in the hydrodynamic radius of the polyplexes at $25{ }^{\circ} \mathrm{C}(48: 6<48: 10<48: 20$; Table 2$)$. This trend may be explained by the molecular weight increase and also by an enhancement of the repulsive electrostatic interactions leading to the complex expansion. However, the smaller hydrodynamic diameter of complexes formed with the highest molecular weight copolymer $(65: 20)$ as compared to the complexes formed with 48:20 suggests that the temperature-responsive block also plays a role in that physical parameter. Furthermore, the fact that this temperatureresponsive contraction is observed at $25{ }^{\circ} \mathrm{C}$ suggests that the complexation of the polymer with the nucleic acids can shift the 

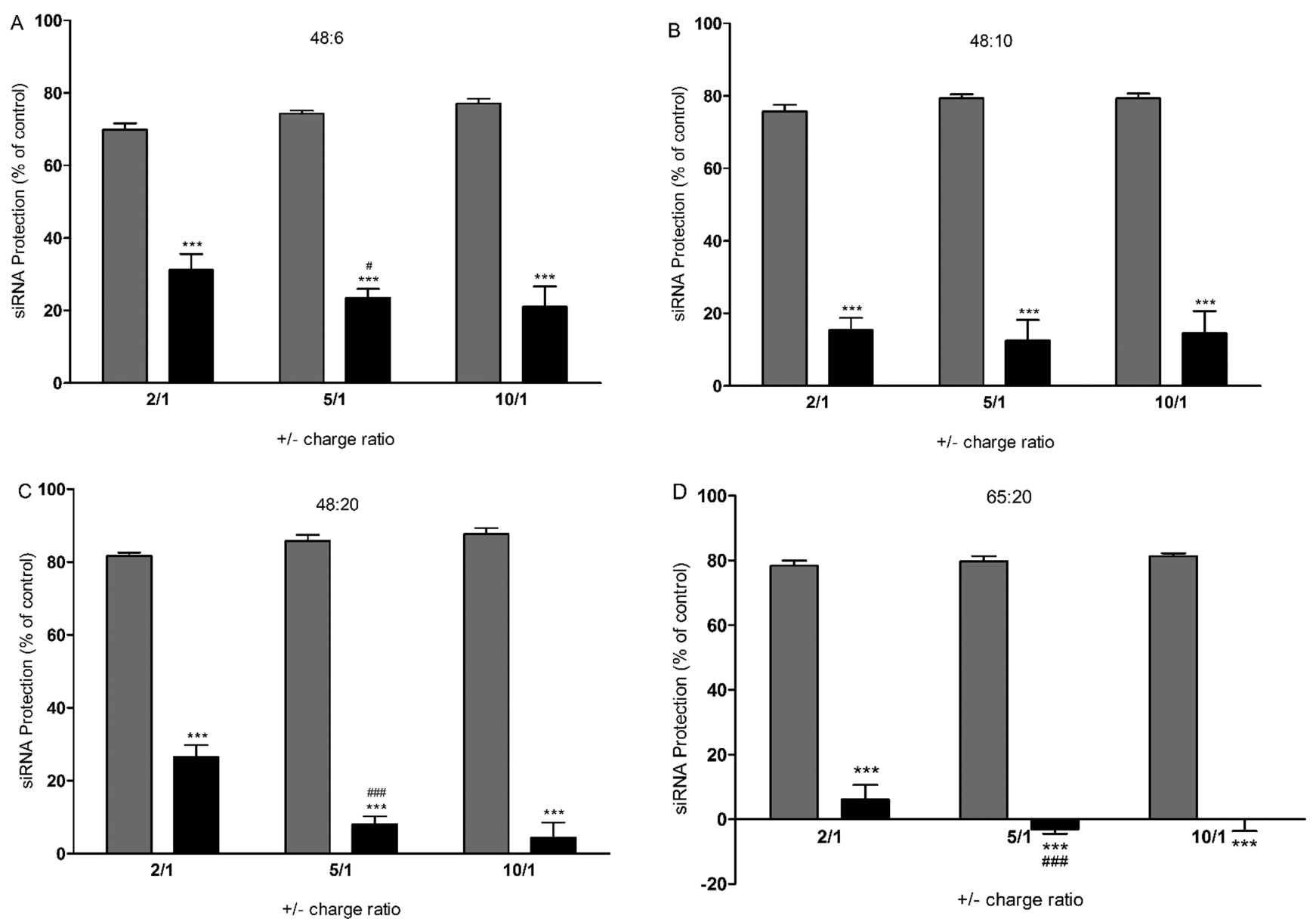

Figure 4. Effect of composition and (+/-) charge ratio of PNIPAAM- $b$-PAMPTMA-based polyplexes on siRNA protection, as assessed by measurement of the PicoGreen fluorescence at $25^{\circ} \mathrm{C}$ (gray) and at $37{ }^{\circ} \mathrm{C}$ (black). Polyplexes, prepared by incubating the copolymers $n: m 48: 6$ (A), 48:10 (B), 48:20 (C), and 65:20 (D) with 14 pmol of siRNA, were added to a PicoGreen solution, as described in the experimental section. The results represent the mean $\pm S D$ obtained from triplicates of three independent experiments. The degree of protection conferred by each polyplex formulation to siRNA at $37^{\circ} \mathrm{C}$ was compared to that conferred by the same formulation at $25^{\circ} \mathrm{C}(* * * p<0.001)$ and to that conferred by the same formulation at the immediately precedent polymer/siRNA $(+/-)$ charge ratio $\left({ }^{\#} p<0.05\right.$ and $\left.{ }^{\# \#} p<0.001\right)$.

transition temperature of PNIPAAM to lower temperatures, in agreement with the charge neutralization of the copolymer.

The increase of temperature from 25 to $37{ }^{\circ} \mathrm{C}$ apparently did not promote statistically significant differences in the hydrodynamic radii for the polyplexes prepared with 48:6, 48:10, and 65:20 copolymers. However, the cloud point values and the thermoresponsive behavior of the copolymers suggest that structural changes take place as the temperature is increased. Interplay between temperature-induced contraction of the polyplexes (contributing to hydrodynamic diameter decrease) and an increased aggregation number (higher number of associating chains, contributing to hydrodynamic diameter increase) may explain the apparently unchanged hydrodynamic diameter of the polyplexes as the temperature is raised. Furthermore, with the exception of polyplexes based on 48:20 copolymers, the hydrodynamic diameter of the polyplexes increased with the increase of $(+/-)$ charge ratio, reflecting an increase of the aggregation number, caused by a higher number of polymer molecules in solution. Interestingly, at $37{ }^{\circ} \mathrm{C}$ the hydrodynamic diameter of the polyplexes prepared with 48:20 copolymers was found to be reduced as the charge ratio increased. This suggests a significant degree of electrostatic repulsion between the complexes formed with the long PAMPTMA $(+)$ block. In the 65:20-containing complexes the electrostatic repulsions are minimized by an extremely long PNIPAAM block.

In a therapeutic context, a siRNA delivery system must be able to carry the nucleic acid molecules to target cells, preventing their degradation by extracellular nucleases, and promote their cellular internalization and release into the cytosol. Because of the inhibitory effect exerted by serum on transfection mediated by nonviral systems, nucleic acid delivery is usually performed in the absence of serum. However, the presence of serum proteins is unavoidable under in vivo conditions. Therefore, in the present work, aiming at predicting the in vivo behavior of the generated PNIPAAM $_{n}-b$ $\operatorname{PAMPTMA}(+)_{m}$-based polyplexes toward their successful clinical application, the knockdown efficiency was evaluated in the presence of serum.

The presence of serum during cell incubation with the complexes caused a mild decrease in their biological activity, as compared to that observed in the absence of serum. This may reflect an unspecific interaction between the cationic moiety of the PNIPAAM- $b$-PAMPTMA(+) copolymers and the negatively charged serum proteins, thus preventing some complexes to reach the cells, and thereby decreasing the knockdown levels. Moreover, the silencing efficiency achieved in the presence of serum was less specific than in its absence, 
indicating the occurrence of off-target effects. The unspecific silencing should be avoided as it can lead to gene mutations and cell transformation, with severe consequences, such as impairment of the cellular machinery responsible for the production of important cell survival proteins. ${ }^{27,28}$

The cloud point of the polymers (Table 1) showed to influence both cytotoxicity and silencing efficiency. In this regard, at the highest polymer/siRNA charge ratios tested, the cytotoxicity induced by the complexes was inversely correlated with the cloud point of the polymers. Both in the absence and presence of $10 \%$ serum, the polymer with the lowest cloud point $(n: m$ 65:20) originated complexes that were highly toxic to the cells. Similar observations have been made when this polymer was used as a carrier for plasmid DNA, and this was then associated with the formation of a compact structure decorated with a highly charged corona. ${ }^{17}$ However, the knockdown efficiency of the polyplexes at the $10 / 1(+/-)$ charge ratio in the absence of serum was found to be also inversely correlated with the polymer transition temperature. Indeed, the silencing activity was most effective for complexes formed by the polymer with the lowest cloud point, with a progressive increase in silencing efficiency according to the following order: 48:20 < 48:10 < 48:6 < 65:20 (Figure 3). This can be explained by the effect of the siRNAs on the cloud point of the copolymers. Because of their negative charges, siRNA molecules may change the ionic strength of the environment or neutralize the charges of the cationic units of the copolymer (which are responsible for increasing the LCST), thus altering the thermal behavior of the complexes, ${ }^{10}$ and eventually promoting their dissociation, by contraction of the polymer in a coil-to-globule transition. ${ }^{29}$

Most cationic systems used to deliver nucleic acids to cells present a protection pattern to siRNA that is consistent with the amount of the cationic moiety employed to formulate the complexes, increasing from the lowest to the highest charge ratios. ${ }^{30,31}$ However, the thermoresponsive copolymers studied herein did not present such behavior. Apparently, the polymer/ siRNA charge ratio did not affect the release of the siRNAs or showed an opposite effect to that described for nonthermoresponsive systems (Figure 4). In general, at $25{ }^{\circ} \mathrm{C}$ (temperature of complex preparation), a very efficient complexation was achieved, and the siRNAs were highly shielded, similarly to what has been described for other cationic polymers used as delivery systems. ${ }^{30}$ However, at $37{ }^{\circ} \mathrm{C}$, the siRNA protection decreased, as the polymers collapsed and dissociated from the nucleic acids. This suggests that at this temperature the increase in polymer concentration mostly results in enhanced intra- and intermolecular hydrophobic associations between the PNIPAAM blocks, contributing to the formation of aggregated and compact structures. In other words, the thermoresponsive properties of the copolymers are dominant in the system, and an increase in charge ratio mostly contributes to increase the associative behavior of PNIPAAM, rather than contributing to protect the siRNA. In this context, the polymer cloud point may also have guided the efficacy of siRNA release, as previously discussed. However, the polymer molecular weight and the number of charged PAMPTMA units determine the number of polymer molecules needed in each case to reach the desired complex charge ratio. Therefore, the organization of the polymeric moieties around the siRNAs was probably sufficiently diverse to lead to the observed differential behavior regarding the nucleic acid release.
In spite of the much lower extent of internalization detected by confocal microscopy, the $n: m 48: 20$ polyplexes, at $10 / 1$ $(+/-)$ charge ratio, showed a relatively high level of GFP knockdown (66\%) as compared to that induced by $n: m$ 48:6 complexes (85\%), which should be attributed to their efficiency in promoting siRNA release. Parallel silencing experiments using lipofectamine RNAi max revealed that the $n: m$ 48:20 copolymer is as efficient as this commercial reagent (65\%) in promoting gene knockdown. Interestingly, the polyplexes formulated with the 48:20 copolymer, at the 10/1 (+/-) charge ratio, were those presenting, at this charge ratio, the largest hydrodynamic diameter at $25{ }^{\circ} \mathrm{C}(>580 \mathrm{~nm})$ and were the smallest $(140 \mathrm{~nm})$ at $37{ }^{\circ} \mathrm{C}$. If, on the one hand, the large hydrodynamic diameter at $25{ }^{\circ} \mathrm{C}$ might be associated with a high siRNA load, the decay in size at $37^{\circ} \mathrm{C}$, on the other hand, can reflect a collapse of the structure upon heating, promoting the release of the entrapped nucleic acids, as described for other drugs. ${ }^{10}$ Because of their small size, the siRNA molecules are exposed after contraction of the copolymers at high temperatures, and their subsequent release by the temperature-induced contraction of the copolymers favors the gene silencing efficiency.

The general picture that emerges from this work is that the silencing efficacy of the studied polyplexes depends on a delicate interplay between repulsive electrostatic forces and hydrophobic interactions, whose balance can be tuned by changing the length of the PNIPAAM or PAMPTMA blocks, which, in turn, defines polymer transition temperature and charge density. The cloud point of the polymer showed, as expected, high relevance for the biological activity achieved upon interaction of the complexes with the cells. It should be stressed that, in the present work, two promising siRNA delivery systems were generated with the $n: m$ 48:6 and $n: m$ 48:20 copolymers and siRNA at the $10 / 1(+/-)$ charge ratio, which revealed high efficiency in silencing a reporter gene in a fibrosarcoma cell line, even in the presence of serum. Under these conditions, the formulation containing $n: m$ 48:20 combines the best relationship between a relatively low-toxic (65\% of cell viability) and highly efficient gene silencing system, with $68 \%$ of GFP knockdown and only $33 \%$ of unspecific gene silencing. Although the size and nucleic acid protection levels of the complexes may limit their application for intravenous administration, the complexes under study can be useful for local delivery into target cells. ${ }^{32}$

Overall, this study opens new trends for the engineering of innovative therapeutic delivery systems from the combination of materials whose properties can synergistically contribute to enhance the efficacy of nucleic acid delivery. With a growing understanding of polymeric drug delivery mechanisms, this approach may be therefore of great relevance to generate new and promising systems for gene therapies.

\section{AUTHOR INFORMATION}

\section{Corresponding Authors}

*(A.S.J.) E-mail: asjurado@bioq.uc.pt.

*(B.N.) E-mail: b.o.g.nystrom@kjemi.uio.no.

\section{Notes}

The authors declare no competing financial interest.

\section{ACKNOWLEDGMENTS}

This work was supported by the Portuguese Foundation for Science and Technology and FEDER/COMPETE (research 
grants PTDC/QUI-BIQ/103001/2008, PTDC/DTP-FTO/ 0265/2012, and Pest-C/SAU/LA0001/2013-2014) and by the Norwegian Research Council, Project Number 190403. A.M.C., C.M.M, and A.L.C. are recipients of fellowships from the Portuguese Foundation for Science and Technology (SFRH/BD/63288/2009, SFRH/BD/79077/2011, and SFRH/BPD/46228/2008, respectively).

\section{REFERENCES}

(1) Brower, V. RNA interference advances to early-stage clinical trials. J. Natl. Cancer Inst. 2010, 102, 1459-1461.

(2) Elbashir, S. M.; Harborth, J.; Lendeckel, W.; Yalcin, A.; Weber, K.; Tuschl, T. Duplexes of 21-nucleotide RNAs mediate RNA interference in cultured mammalian cells. Nature 2001, 411, 494-8.

(3) Jinek, M.; Doudna, J. A. A three-dimensional view of the molecular machinery of RNA interference. Nature 2009, 457, 405-12.

(4) Wu, S. Y.; McMillan, N. A. J. Lipidic systems for in vivo siRNA delivery. AAPS J. 2009, 11, 639-52.

(5) Gavrilov, K.; Saltzman, W. M. Therapeutic siRNA: Principles, challenges, and Strategies. Yale J. Biol. Med. 2012, 85, 187-200.

(6) Troiber, C.; Wagner, E. Nucleic acid carriers based on precise polymer conjugates. Bioconjugate Chem. 2011, 22, 1737-52.

(7) Meade, B. R.; Dowdy, S. F. Enhancing the cellular uptake of siRNA duplexes following noncovalent packaging with protein transduction domain peptides. Adv. Drug Delivery Rev. 2008, 60, $530-6$.

(8) Schild, H. G. Poly( $N$-isopropylacrylamide): experiment, theory and application. Prog. Polym. Sci. 1992, 17, 163-249.

(9) Masci, G.; Ladogana, R. D.; Cametti, C. Assemblies of thermoresponsive diblock copolymers: micelle and vesicle formation investigated by means of dielectric relaxation spectroscopy. J. Phys. Chem. B 2012, 116, 2121-30.

(10) Ward, M. A.; Georgiou, T. K. Thermoresponsive Polymers for Biomedical Applications. Polymers 2011, 3, 1215-1242.

(11) Jain, S.; Bates, F. S. On the origins of morphological complexity in block copolymer surfactants. Science 2003, 300, 460-4.

(12) Pamies, R.; Zhu, K.; Kjøniksen, A.-L.; Nyström, B. Thermal response of low molecular weight poly-( $N$-isopropylacrylamide $)$ polymers in aqueous solution. Polym. Bull. 2009, 62, 487-502.

(13) Theato, P.; Sumerlin, B. S.; O’Reilly, R. K.; Epps, T. H., III. Stimuli responsive materials. Chem. Soc. Rev. 2013, .

(14) Alexander, C. Temperature- and $\mathrm{pH}$-responsive smart polymers for gene delivery. Expert Opin. Drug Delivery 2006, 3, 573-581.

(15) Guo, J.; Fisher, K. A; Darcy, R.; Cryan, J. F.; O’Driscoll, C. Therapeutic targeting in the silent era: advances in non-viral siRNA delivery. Mol. BioSyst. 2010, 6, 1143-61.

(16) Takeda, N.; Nakamura, E.; Yokoyama, M.; Okano, T. Temperature-responsive polymeric carriers incorporating hydrophobic monomers for effective transfection in small doses. J. Controlled Release 2004, 95, 343-55.

(17) Calejo, M. T.; Cardoso, A. M. S.; Kjøniksen, A.; Zhu, K.; Morais, C. M.; Sande, S. A.; Cardoso, A. L.; Pedroso de Lima, M. C.; Jurado, A.; Nyström, B. Temperature-responsive cationic block copolymers as nanocarriers for gene delivery. Int. J. Pharm. 2013, 448, 105-114.

(18) Kim, C.; Lee, Y.; Kim, J. S.; Jeong, J. H.; Park, T. G. Thermally triggered cellular uptake of quantum dots immobilized with poly $(\mathrm{N}$ isopropylacrylamide) and cell penetrating peptide. Langmuir 2010, 26, 14965-9.

(19) Caplen, N. J. Gene therapy progress and prospects. Downregulating gene expression: the impact of RNA interference. Gene Ther. 2004, 11, 1241-8.

(20) Fire, A.; Xu, S.; Montgomery, M. K.; Kostas, S. A.; Driver, S. E.; Mello, C. C. Potentandspecific genetic interference by double-stranded RNA in Caenorhabditis elegans. Nat. Lett. 1998, 391, 806-811.

(21) Hannon, G. J.; Rossi, J. J. Unlocking the potential of the human genome with RNA interference. Nature 2004, 431, 371-8.

(22) Napoli, C.; Lemieux, C.; Jorgensen, R. Introduction of a chimeric chalcone synthase gene into petunia results in reversible co- suppression of homologous genes in trans. Plant Cell 1990, 2, 279289.

(23) Dedinaite, A.; Thormann, E.; Olanya, G.; Claesson, P. M.; Nyström, B.; Kjøniksen, A.-L.; Zhu, K. Friction in aqueous media tuned by temperature-responsive polymer layers. Soft Matter 2010, 6, 2489-98.

(24) Kjøniksen, A.-L.; Zhu, K.; Karlsson, G.; Nyström, B. Novel transition behavior in aqueous solutions of a charged thermoresponsive triblock copolymer. Colloids Surf., A 2009, 333, 32-45.

(25) Cardoso, A.; Trabulo, S.; Moreira, J. N.; Duzgunes, N.; Pedroso de Lima, M. C. Targeted Lipoplexes for siRNA Delivery. Methods Enzymol. 2009, 465, 267-287.

(26) Simões, S.; Slepushkin, V.; Pires, P.; Gaspar, R.; de Lima, M. P.; Düzgüneş, N. Mechanisms of gene transfer mediated by lipoplexes associated with targeting ligands or $\mathrm{pH}$-sensitive peptides. Gene Ther. 1999, 6, 1798-807.

(27) Birmingham, A.; Anderson, E. M.; Reynolds, A.; Ilsley-Tyree, D.; Leake, D.; Fedorov, Y.; Baskerville, S.; Maksimova, E.; Robinson, K.; Karpilow, J.; Marshall, W. S.; Khvorova, A. 3' UTR seed matches, but not overall identity, are associated with RNAi off-targets. Nat. Methods 2006, 3, 199-204.

(28) Jackson, A. L.; Burchard, J.; Schelter, J.; Chau, B. N.; Cleary, M.; Lim, L.; Linsley, P. S. Widespread siRNA "off-target" transcript silencing mediated by seed region sequence complementarity. RNA 2006, 12, 1179-1187.

(29) Volden, S.; Ese, M.-H. G.; Zhu, K.; Yasuda, M.; Nyström, B.; Glomm, W. R. Interactions between bovine serum albumin and Langmuir films composed of charged and uncharged poly $(\mathrm{N}$ isopropylacrylamide) block copolymers. Colloids Surf., B 2012, 98, 50-7.

(30) Crombez, L.; Aldrian-Herrada, G.; Konate, K.; Nguyen, Q. N.; McMaster, G. K.; Brasseur, R; Heitz, F.; Divita, G. A new potent secondary amphipathic cell-penetrating peptide for siRNA delivery into mammalian cells. Mol. Ther. 2009, 17, 95-103.

(31) Tsai, L.-R.; Chen, M.-H.; Chien, C.-T.; Chen, M.-K.; Lin, F.-S.; Lin, K. M.-C.; Hwu, Y.-K.; Yang, C.-S.; Lin, S.-Y. A single-monomer derived linear-like PEI-co-PEG for siRNA delivery and silencing. Biomaterials 2011, 32, 3647-53.

(32) Hrubya, M.; Pouckova, P.; Zadinova, M.; Kuckaa, J.; Lebeda, O. Thermoresponsive polymeric radionuclide delivery system: An injectable brachytherapy. Eur. J. Pharm. Sci. 2011, 42, 484-488. 